\title{
RETRACTED ARTICLE: The diagnostic performance of 18F-FDG PET in detecting bone marrow infiltration in Hodgkin's lymphoma and diffuse large B-cell lymphoma: a meta-analysis
}

\author{
Budong Chen • Dan Han • Benjamin H. Ge $\cdot$ Chunlin Li • \\ Daqing Ma $\cdot$ Jigang Yang
}

Received: 18 May 2014 / Revised: 18 May 2014 / Accepted: 19 August 2014 /Published online: 7 September 2014

(C) ISS 2014

Upon request of the authors, this article is retracted because of substantial overlap with language published in "FDG PET/CT for the detection of bone marrow involvement in diffuse large B-celllymphoma: systematic review and meta-analysis (Hugo J. A. Adams, Thomas C. Kwee, Bart de Keizer,Rob Fijnheer, John M. H. de Klerk, Rutger A. J. Nievelstein.; Eur J Nucl Med Mol Imaging (2014)41:565-574”.

\footnotetext{
Upon request of the authors, this article is retracted because of substantial overlap with language published in "FDG PET/CT for the detection of bone marrow involvement in diffuse large B-celllymphoma: systematic review and meta-analysis (Hugo J. A. Adams, Thomas C. Kwee, Bart de Keizer,Rob Fijnheer, John M. H. de Klerk, Rutger A. J. Nievelstein.; Eur J Nucl Med Mol Imaging (2014)41:565-574".
}

B. Chen $\cdot$ D. Han $\cdot$ D. Ma

Radiology Department, Beijing Friendship Hospital of Capital

Medical University, Beijing 100050, China

B. H. Ge

Department of Radiology, Hospital of the University of

Pennsylvania, Philadelphia, PA 19104, USA

C. Li $\cdot$ J. Yang $(\triangle)$

Nuclear Medicine Department, Beijing Friendship Hospital of Capital Medical University, 95 Yong An Road, Xi Cheng District, Beijing 100050, China

e-mail: nmyangjigang@gmail.com 